\title{
Theoretic properties of the symmetric algebra of edge ideals
}

\author{
Maurizio Imbesi Monica La Barbiera
}

\begin{abstract}
In this note the integrality and the reducedness for the symmetric algebra of the edge ideals of simple graphs are analyzed. More precisely, we will extend a result on the integrality of the symmetric algebra given for connected graphs, stating a characterization for any simple graph. Moreover, through the Gröbner bases theory, we investigate simple graphs for which the symmetric algebra is reduced, in particular we prove its reducedness for graphs with $n$ vertices consisting of a cycle of length $n$.
\end{abstract}

\section{Introduction}

Let $\mathcal{G}$ be a simple graph, $V(\mathcal{G})=\left\{v_{1}, \ldots, v_{n}\right\}$ be the set of the vertices of $\mathcal{G}$, and $E(\mathcal{G})$ be the sets of its edges.

Let $R=k\left[X_{1}, \ldots, X_{n}\right]$ be the polynomial ring in $n$ variables, $k$ a field, such that the indeterminate $X_{i}$ of $R$ is associated to the vertex $v_{i}$ of $\mathcal{G}$. The edge ideal $I(\mathcal{G})$ of $\mathcal{G}$ is a monomial ideal of $R$ generated by squarefree monomials of degree 2 which correspond to the edges of $\mathcal{G}$, namely $I(\mathcal{G})=\left(X_{i} X_{j} \mid\left\{v_{i}, v_{j}\right\}\right.$ is an edge of $\left.\mathcal{G}\right)$.

In [12], fundamental results about monomial ideals of $R$ that can arise from the edges of a simple graph $\mathcal{G}$ can be found.

In the present paper we are interested to study some important algebraic properties of the symmetric algebra $S_{R}(I(\mathcal{G}))$ of the edge ideal of $\mathcal{G}$.

The symmetric algebra of $I(\mathcal{G})$ over $R$ is defined to be

$$
S_{R}(I(\mathcal{G}))=R\left[Y_{i j} \mid\left\{v_{i}, v_{j}\right\} \in E(\mathcal{G})\right] / J,
$$

Received by the editors in November 2013.

Communicated by M. Van den Bergh.

2010 Mathematics Subject Classification : 13A02, 13A30, 13P10, 15A78.

Key words and phrases : Edge ideals, symmetric algebra, Gröbner bases. 
where $J$ is the ideal of relations of $S_{R}(I(\mathcal{G}))$, namely the binomial ideal

$$
\begin{aligned}
J= & \left(X_{i} Y_{j k}-X_{j} Y_{i k} \mid\left\{v_{j}, v_{k}\right\},\left\{v_{i}, v_{k}\right\} \in E(\mathcal{G})\right)+ \\
& +\left(X_{i} X_{j} Y_{\ell k}-X_{\ell} X_{k} Y_{i j} \mid\left\{v_{\ell}, v_{k}\right\} \cap\left\{v_{i}, v_{j}\right\}=\varnothing\right) .
\end{aligned}
$$

In [5] it is examined the projective dimension of the edge ideals of simple graphs with respect to the number of the edges of such graphs and consequences of it. The symmetric algebra of the edge ideals of simple graphs is also studied in order to give conditions for which it is a integrity domain. More precisely, results about the integrality of the symmetric algebra are obtained for simple graphs with at most three edges and for Cohen-Macaulay graphs without cycles of even length and vertex covering number 3.

Classes of edge ideals of linear type, i.e. ideals whose symmetric algebra is a integrity domain, are described in [7]. Computations of standard algebraic invariants for the symmetric algebras of significative edge ideals which are generated by $s$-sequences are made in $[1,6]$.

Here we give a complete characterization about the integrality of the symmetric algebra of the edge ideal of simple graphs and investigate the reducedness of such an algebra.

The paper is structured as follows.

In section 1 some useful definitions and properties involving algebra, graph theory and combinatorics are introduced.

In section 2 we examine general simple graphs and study the integrality of the symmetric algebra of their edge ideal. In [13], it is proved a result that examines the connected simple graphs whose edge ideals are of linear type. The main theorem of this section extends to all simple graphs the foregoing characterization. Its terms are the following

Let $\mathcal{G}$ be any graph, $I(\mathcal{G})$ be its edge ideal. The symmetric algebra $S_{R}(I(\mathcal{G}))$ is a integrity domain if and only if every connected subgraph of $\mathcal{G}$ has either at most a cycle of odd length or no cycle of even length.

In section 3, using the Gröbner bases theory, we intend to investigate the reducedness of the symmetric algebra of the edge ideal of simple graphs $\mathcal{G}$. We prove a necessary condition, involving the $S$-polynomials, in order that the symmetric algebra of $I(\mathcal{G})$ is reduced. However, for the graphs $\mathcal{C}_{n}$ consisting of a cycle of length $n$, we show that the elements of a Gröbner basis of the ideal of relations $J$ of $S_{R}\left(I\left(\mathcal{C}_{n}\right)\right)$ have squarefree initial terms, then the initial ideal of $J$ is squarefree, and consequently the symmetric algebra is reduced. This seems to bring forward good reasons for conjecturing that $S_{R}(I(\mathcal{G}))$ is reduced when $\mathcal{G}$ is any graph with at least a cycle of even length.

\section{Preliminaries and notations}

Let $\mathcal{G}$ be a graph, $V(\mathcal{G})$ and $E(\mathcal{G})$ be the sets of its vertices and edges, respectively. $\mathcal{G}$ is said to be simple or loopless if, for all $\left\{v_{i}, v_{j}\right\} \in E(\mathcal{G}), i \neq j$, it is $v_{i} \neq v_{j}$. A walk of length $r$ in $\mathcal{G}$ is an alternating sequence of vertices and edges $\left\{v_{0}, l_{1}, v_{1}, l_{2}, \ldots, v_{r-1}, l_{r}, v_{r}\right\}$, where $l_{i}=\left\{v_{i-1}, v_{i}\right\} \in E(\mathcal{G})$. A walk is said closed if $v_{0}=v_{r}$. A walk may also be denoted by $\left\{v_{0}, \ldots, v_{r}\right\}$, the edges being clear by context. 
A cycle of length $r$ is a closed walk in which the vertices $v_{1}, \ldots, v_{r}$ are distinct. We denote by $\mathcal{C}_{n}$ the graph with $n$ vertices consisting of a cycle of length $n$. A cycle of even length will be called a monomial walk.

A tree is a connected graph without cycles.

The degree of a vertex $v_{h} \in V(\mathcal{G})$, indicated by $\operatorname{deg}\left(v_{h}\right)$, is the number of edges incident with $v_{h}$, i.e. $\operatorname{deg}\left(v_{h}\right)=\max \left\{s \mid \exists v_{1}, \ldots, v_{s} \in V(\mathcal{G})\right.$ with $\left\{v_{i}, v_{h}\right\} \in E(\mathcal{G})$, $i=1, \ldots, s\}$. When $\operatorname{deg}\left(v_{h}\right)=0$, the vertex $v_{h}$ is said isolated.

A subset $C$ of $V(\mathcal{G})$ is said a minimal vertex cover for $\mathcal{G}$ if every edge of $\mathcal{G}$ is incident with one vertex in $C$ and there is no proper subset of $C$ having such a property. If $C$ satisfies the incident condition only, it is called a vertex cover for $\mathcal{G}$.

The smallest number of vertices in any minimal vertex cover for $\mathcal{G}$ is called vertex covering number of $\mathcal{G}$ and denoted by $\alpha_{0}(\mathcal{G})$.

If $V(\mathcal{G})=\left\{v_{1}, \ldots, v_{n}\right\}$ and $R=k\left[X_{1}, \ldots, X_{n}\right]$ such that each variable $X_{i}$ of $R$ corresponds to the vertex $v_{i}$ of $\mathcal{G}$, the edge ideal $I(\mathcal{G})$ of $\mathcal{G}$ is the ideal $\left(X_{i} X_{j} \mid\left\{v_{i}, v_{j}\right\} \in\right.$ $E(\mathcal{G})) \subset R$.

Note that the non-zero edge ideals are those generated by squarefree monomials of degree 2. This implies that $I(\mathcal{G})$ is a graded ideal of $R$ of initial degree 2 , that is $I(\mathcal{G})=\oplus_{i \geqslant 2}\left(I(\mathcal{G})_{i}\right)$.

$I(\mathcal{G})=(0)$ if and only if $\mathcal{G}$ has isolated vertices only.

So $I(\mathcal{G})$ has a graded free resolution of length at most $n$. The length of the (unique) minimal resolution of $I(\mathcal{G})$ is equal to $\operatorname{pd}_{R}(I(\mathcal{G})$ ), the projective dimension of $I(\mathcal{G})$.

An ideal $\wp \subset R$ generated by $C=\left\{X_{i_{1}}, \ldots, X_{i_{s}}\right\}$ is a minimal prime ideal of $I(\mathcal{G})$ if and only if $C$ is a minimal vertex cover for $\mathcal{G}$.

From this, it descends $\alpha_{0}(\mathcal{G})=\operatorname{ht}(I(\mathcal{G})$ ) ([12], Corollary 6.1.18).

$\mathcal{G}$ is said a Cohen-Macaulay graph over $k$ if $\operatorname{depth}(R / I(\mathcal{G}))=\operatorname{dim}(R / I(\mathcal{G}))$, where dim is the Krull dimension.

If $\mathcal{G}$ is a Cohen-Macaulay graph, the Auslander-Buchsbaum theorem ([3], Corollary 3.3) gives $\operatorname{pd}_{R}(I(\mathcal{G}))=\alpha_{0}(\mathcal{G})-1$.

Definition 1.1. Let $I$ be an ideal of $R=k\left[X_{1}, \ldots, X_{n}\right]$. The symmetric algebra of $I$ over $R$ is the graded algebra $S_{R}(I)=\bigoplus_{t \geqslant 0} S_{t}(I)$, where $S_{t}(I)$ are the symmetric powers.

Let $R^{p} \stackrel{\varphi}{\longrightarrow} R^{q} \stackrel{\psi}{\longrightarrow} I \rightarrow 0$ be a presentation of $I$, where $\varphi=\left(a_{i j}\right)$ is a $q \times p$ matrix with entries in $R$. Then $S_{R}(I) \simeq R\left[Y_{1}, \ldots, Y_{q}\right] / J$, where $J=\left(g_{1}, \ldots, g_{p}\right)$ denotes the ideal of relations of $S_{R}(I)$, and $g_{j}=\sum_{i, j} a_{i j} Y_{i}$.

For further notions and basic properties of such an algebra, see [9].

Now, let $I(\mathcal{G}) \subset R=k\left[X_{1}, \ldots, X_{n}\right]$ be the edge ideal of the graph $\mathcal{G}$. The symmetric algebra of $I(\mathcal{G})$ can be written

$$
S_{R}(I(\mathcal{G}))=R\left[Y_{i j} \mid\left\{v_{i}, v_{j}\right\} \in E(\mathcal{G})\right] / J,
$$

where the ideal of relations $J$ of $S_{R}(I(\mathcal{G}))$ is defined by $J=\left(X_{i} Y_{j k}-X_{j} Y_{i k} \mid\right.$ $\left.\left\{v_{j}, v_{k}\right\},\left\{v_{i}, v_{k}\right\} \in E(\mathcal{G})\right)+\left(X_{i} X_{j} Y_{\ell k}-X_{\ell} X_{k} Y_{i j} \mid\left\{v_{\ell}, v_{k}\right\} \cap\left\{v_{i}, v_{j}\right\}=\varnothing\right)$.

Definition 1.2. Let $R$ be any ring. The set of all nilpotent elements of $R$ is called nilradical of $R$ and it is denoted by $\operatorname{Nil}(R)$. 
Remark 1.1. (See [8])

The set $\operatorname{Nil}(R)=\sqrt{(0)}$, where $\sqrt{(0)}=\left\{x \in R \mid x^{n} \in(0)\right.$ for some $\left.n>0\right\}$ is the radical of the ideal (0). For every ideal $I$ of $R$ one has that the radical of $I$ is the intersection of all prime ideals containing $I$. Hence $\operatorname{Nil}(R)$ is the intersection of all prime ideals of $R$.

Recall that an algebra is said to be reduced if its nilradical is $(0)$.

\section{Integrality of the symmetric algebra of edge ideals}

In this section we examine simple graphs and study the integrality of the symmetric algebra of their edge ideal.

In [4] it is shown that the symmetric algebra for meaningful classes of simple graphs is a integrity domain. In particular, for simple graphs with at most three edges, the following are established.

Proposition 2.1. Let $I(\mathcal{G})$ be the edge ideal of a simple graph $\mathcal{G}$ such that $|E(\mathcal{G})|<4$. Then the symmetric algebra $S_{R}(I(\mathcal{G}))$ is a integrity domain.

Proof. See [4], Proposition 3.1 .

Corollary 2.2. Let $\mathcal{G}$ be a simple graph such that $\operatorname{pd}_{R}(I(\mathcal{G})) \leqslant 1$. Then the symmetric algebra $S_{R}(I(\mathcal{G}))$ is a integrity domain.

In the same work, further results for Cohen-Macaulay graphs without monomial walks and vertex covering number 3 are given.

Proposition 2.3. Let $\mathcal{G}$ be a Cohen-Macaulay graph without monomial walks and $\operatorname{pd}_{R}(I(\mathcal{G}))=2$. Then the symmetric algebra $S_{R}(I(\mathcal{G}))$ is a integrity domain.

Proof. See [4], Proposition 3.3.

Remark 2.4. If the symmetric algebra of $I(\mathcal{G})$ in $R$ is a integrity domain, then the canonical morphism of graded algebras

$$
S(I(\mathcal{G})) \longrightarrow \mathcal{R}(I(\mathcal{G}))
$$

is an isomorphism, where $\mathcal{R}(I(\mathcal{G}))=\bigoplus_{k \geqslant 0} I(\mathcal{G})^{k}=R[I(\mathcal{G}), t] \subset R[t]$ is the Rees algebra of $I(\mathcal{G})$ in $R$.

In this case $I(\mathcal{G})$ is said to be of linear type.

Corollary 2.5. Let $\mathcal{G}$ be a Cohen-Macaulay graph without monomial walks and $\alpha_{0}(\mathcal{G})=3$. Then the edge ideal $I(\mathcal{G})$ is of linear type.

Corollary 2.6. Let $\mathcal{G}$ be a Cohen-Macaulay graph with $|E(\mathcal{G})| \leqslant 6$ without monomial walks. Then $I(\mathcal{G})$ is of linear type. 
An important result about the integrality of the symmetric algebra is

Proposition 2.7. (See [10])

Let $R$ be a domain, $M$ be a finitely generated $R$-module. Then the symmetric algebra $S_{R}(M)$ is a domain if and only if every symmetric power $S_{t}(M)$ is torsion free, for all $t \geqslant 0$.

For an ideal $I$ of a domain $R$ it is known

Proposition 2.8. (See [10])

Let $R$ be a domain and $I$ be an ideal of R. TFAE:

1) $S_{R}(I)$ is a integrity domain;

2) $S_{R}(I)$ is torsion free;

3) $S_{R}(I) \cong \Re(I)$, where $\Re(I)$ is the Rees algebra of $I$.

Note that the above condition 3) is equivalent to say that $I$ is of linear type.

In [13], for a connected graph, the following fact is stated.

Proposition 2.9. Let $\mathcal{G}$ be a connected graph and $I(\mathcal{G})$ be its edge ideal. Then $I(\mathcal{G})$ is an ideal of linear type if and only if $\mathcal{G}$ is a tree or $\mathcal{G}$ has a unique cycle of odd length.

Now we will extend last result by establishing a characterization for any simple graph. First introduce some notions.

Definition 2.1. Let $R=k\left[X_{1}, \ldots, X_{n}\right]$. If $m=X_{1}^{a_{1}} \cdots X_{n}^{a_{n}}$ is a monomial in $R$ we set:

1) $\operatorname{supp}(m)=\left\{X_{i} \mid a_{i}>0\right\}$;

2) ${ }^{*} \operatorname{supp}(m)=\{\underbrace{X_{1}, \ldots, X_{1}}_{a_{1}-\text { times }}, \ldots, \underbrace{X_{n}, \ldots, X_{n}}_{a_{n}-\text { times }}\}$.

Remark 2.10. Let $m, m^{\prime}$ be monomials in $R$. One has: ${ }^{*} \operatorname{supp}(m)={ }^{*} \operatorname{supp}\left(m^{\prime}\right) \Leftrightarrow$ $m=m^{\prime}$. Indeed, $\operatorname{supp}(m)=\operatorname{supp}\left(m^{\prime}\right) \nRightarrow m=m^{\prime}$.

Let $E_{i}$ be a subset of $E(\mathcal{G})$. We set $\overline{E_{i}}=\bigcup_{\left\{v_{i}, v_{j}\right\} \in E_{i}}\left\{X_{i}, X_{j}\right\}$.

Example 2.11. Let $\mathcal{G}$ be the graph $C_{4}$. Let $E_{1}=\left\{X_{1} X_{2}, X_{3} X_{4}\right\}$ and $E_{2}=\left\{X_{2} X_{3}\right.$, $\left.X_{1} X_{4}\right\}$. Then $\overline{E_{1}}=\overline{E_{2}}=\left\{X_{1}, X_{2}, X_{3}, X_{4}\right\}$.

Proposition 2.12. Let $\mathcal{G}$ be any graph such that every connected subgraph of $\mathcal{G}$ has either at most a cycle of odd length or no monomial walk. If $E_{1}, E_{2} \subset E(\mathcal{G})$ and $m, m^{\prime}$ are monomials in $R$ such that

(1) ${ }^{*} \operatorname{supp}(m) \cup \overline{E_{1}}={ }^{*} \operatorname{supp}\left(m^{\prime}\right) \cup \overline{E_{2}}$

(2) $\left|E_{1}\right|=\left|E_{2}\right|$,

then

$$
m \prod_{\left\{v_{i}, v_{j}\right\} \in E_{1}} Y_{i j}-m^{\prime} \prod_{\left\{v_{i^{\prime},}, v_{j^{\prime}}\right\} \in E_{2}} Y_{i^{\prime} j^{\prime}} \in J
$$


Proof. We work by induction on $t=\left|E_{1}\right|=\left|E_{2}\right|$.

Let $t=0$. One has ${ }^{*} \operatorname{supp}(m)={ }^{*} \operatorname{supp}\left(m^{\prime}\right)$, then $m-m^{\prime}=0 \in J$.

Suppose $t>0$.

We may assume * $\operatorname{supp}\left(m^{\prime \prime}\right)={ }^{*} \operatorname{supp}(m) \cap{ }^{*} \operatorname{supp}\left(m^{\prime}\right)=\varnothing$, where $m^{\prime \prime}$ is a monomial in $R$ that divides $m$ and $m^{\prime}$. If

$$
\frac{m}{m^{\prime \prime}} \prod_{\left\{v_{i}, v_{j}\right\} \in E_{1}} Y_{i j}-\frac{m^{\prime}}{m^{\prime \prime}} \prod_{\left\{v_{i^{\prime}}, v_{j^{\prime}}\right\} \in E_{2}} Y_{i^{\prime} j^{\prime}} \in J,
$$

then

$$
m \prod_{\left\{v_{i}, v_{j}\right\} \in E_{1}} Y_{i j}-m^{\prime} \prod_{\left\{v_{i^{\prime}}, v_{j^{\prime}}\right\} \in E_{2}} Y_{i^{\prime} j^{\prime}}=m^{\prime \prime}\left(\frac{m}{m^{\prime \prime}} \prod_{\left\{v_{i}, v_{j}\right\} \in E_{1}} Y_{i j}-\frac{m^{\prime}}{m^{\prime \prime}} \prod_{\left\{v_{i^{\prime}}, v_{j^{\prime}}\right\} \in E_{2}} Y_{i^{\prime} j^{\prime}}\right)
$$

belongs to $J$, where ${ }^{*} \operatorname{supp}\left(\frac{m}{m^{\prime \prime}}\right) \cap{ }^{*} \operatorname{supp}\left(\frac{m^{\prime}}{m^{\prime \prime}}\right)=\varnothing$.

Hence we may assume $E_{1} \cap E_{2}=\varnothing$. Moreover it results $m, m^{\prime} \neq 1$. In fact, if $m=m^{\prime}=1$, which implies ${ }^{*} \operatorname{supp}(m)={ }^{*} \operatorname{supp}\left(m^{\prime}\right)=\varnothing$, then by $(1)$ it is $\overline{E_{1}}=\overline{E_{2}}$. By hypothesis, every connected subgraph of $\mathcal{G}$ has either at most a cycle of odd length or no monomial walk, hence a contradiction follows.

Suppose $X_{i} \mid m . \exists j$ such that $\left\{v_{i}, v_{j}\right\} \in E_{2}$, being $m \prod Y_{i j}-m^{\prime} \prod Y_{i^{\prime} j^{\prime}} \in J$.

Then, (i) $\exists v_{k}$ such that $\left\{v_{j}, v_{k}\right\} \in E_{1}$, or

(ii) $\nexists v_{k}$ such that $\left\{v_{j}, v_{k}\right\} \in E_{1}$.

Case (i)

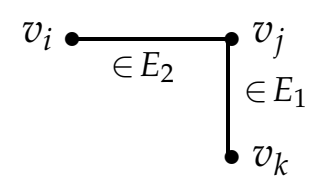

It is

$$
X_{k} Y_{i j}-X_{i} Y_{j k} \in J
$$

By induction hypothesis,

$$
\frac{m}{X_{i}} X_{k} \prod_{\left\{v_{p}, v_{q}\right\} \in E_{1} \backslash\left\{\left\{v_{j}, v_{k}\right\}\right\}} Y_{p q}-m^{\prime} \prod_{\left\{v_{p}, v_{q}\right\} \in E_{2} \backslash\left\{\left\{v_{i}, v_{j}\right\}\right\}} Y_{p q} \in J ，
$$

and multiplying by $Y_{i j}$, it is

$$
\frac{m}{X_{i}} X_{k} Y_{i j} \prod_{\left\{v_{p}, v_{q}\right\} \in E_{1} \backslash\left\{\left\{v_{j}, v_{k}\right\}\right\}} Y_{p q}-m^{\prime} Y_{i j} \prod_{\left\{v_{p}, v_{q}\right\} \in E_{2} \backslash\left\{\left\{v_{i}, v_{j}\right\}\right\}} Y_{p q} \in J .
$$

By (2.1), (2.2),

$$
m \prod_{\left\{v_{p}, v_{q}\right\} \in E_{1}} Y_{p q}-m^{\prime} \prod_{\left\{v_{p}, v_{q}\right\} \in E_{2}} Y_{p q} \in J
$$

Case (ii)

If $\nexists v_{k}$ such that $\left\{v_{j}, v_{k}\right\} \in E_{1}$, then $X_{j} \mid m$. So,

( $\alpha) \exists v_{\ell}$ such that $\left\{v_{i}, v_{\ell}\right\} \in E_{1}$, or

( $\beta) \exists\left\{v_{r}, v_{s}\right\} \in E_{1}$ such that $\left\{v_{r}, v_{s}\right\} \cap\left\{v_{i}, v_{j}\right\}=\varnothing$. 
For $(\alpha) \quad \in E_{1}^{v_{i}} \underset{v_{\ell}}{v_{\ell}} v_{j}$

it is

$$
X_{\ell} Y_{i j}-X_{j} Y_{i \ell} \in J
$$

by induction hypothesis and multiplying by $Y_{i j}$, it is

$$
\frac{m}{X_{j}} X_{\ell} Y_{i j} \prod_{\left\{v_{p}, v_{q}\right\} \in E_{1} \backslash\left\{\left\{v_{i}, v_{\ell}\right\}\right\}} Y_{p q}-m^{\prime} Y_{i j} \prod_{\left\{v_{p}, v_{q}\right\} \in E_{2} \backslash\left\{\left\{v_{i}, v_{j}\right\}\right\}} Y_{p q} \in J .
$$

By (2.3), (2.4),

$$
m \prod_{\left\{v_{p}, v_{q}\right\} \in E_{1}} Y_{p q}-m^{\prime} \prod_{\left\{v_{p}, v_{q}\right\} \in E_{2}} Y_{p q} \in J
$$

For $(\beta)$

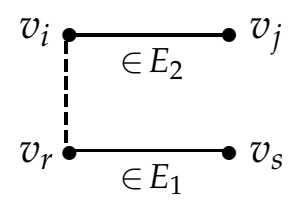

if $\left\{v_{r}, v_{s}\right\}$ and $\left\{v_{i}, v_{j}\right\}$ are disconnected in $\mathcal{G}$, then by definition of $J$, it is

$$
X_{r} X_{s} Y_{i j}-X_{i} X_{j} Y_{r s} \in J \text {; }
$$

if $\left\{v_{r}, v_{s}\right\}$ and $\left\{v_{i}, v_{j}\right\}$ are connected in $\mathcal{G}$, say $\left\{v_{i}, v_{r}\right\} \in E(\mathcal{G})$, then again

$$
X_{r} X_{s} Y_{i j}-X_{i} X_{j} Y_{r s}=X_{s}\left(X_{r} Y_{i j}-X_{j} Y_{i r}\right)+X_{j}\left(X_{s} Y_{i r}-X_{i} Y_{r s}\right) \in J
$$

With the same argument

$$
\frac{m}{X_{i} X_{j}} X_{r} X_{s} Y_{i j} \prod_{\left\{v_{p}, v_{q}\right\} \in E_{1} \backslash\left\{\left\{v_{r}, v_{s}\right\}\right\}} Y_{p q}-m^{\prime} Y_{i j} \prod_{\left\{v_{p}, v_{q}\right\} \in E_{2} \backslash\left\{\left\{v_{i}, v_{j}\right\}\right\}} Y_{p q} \in J .
$$

By (2.5), (2.6),

$$
m \prod_{\left\{v_{p}, v_{q}\right\} \in E_{1}} Y_{p q}-m^{\prime} \prod_{\left\{v_{p}, v_{q}\right\} \in E_{2}} Y_{p q} \in J
$$

Theorem 2.13. Let $\mathcal{G}$ be any graph, $I(\mathcal{G})$ be its edge ideal. The symmetric algebra $S_{R}(I(\mathcal{G}))$ is a integrity domain if and only if every connected subgraph of $\mathcal{G}$ has either at most a cycle of odd length or no monomial walk.

Proof. $\Longrightarrow)$ First let's show that if $\mathcal{G}$ possesses at least a monomial walk, then $S_{R}(I(\mathcal{G}))$ is not a integrity domain.

Without loss of generality, let's reduce to examine the case of a subgraph $\mathcal{H}$ of $\mathcal{G}$ consisting of a cycle of even length $2 k$.

Among the binomials that generate the ideal of relations $J$ of $S_{R}(I(\mathcal{H}))$, let's take the $2 k$ ones having degree 2 and order them with respect to the indices of the vertices of $\mathcal{H}$.

Sum up the $1^{\text {st }}$, the $3^{\text {rd }}, \ldots$, the $(2 k-1)$-th binomials, after multiplying each of them by convenient $k-1$ factors $Y_{i, i+1}, i=1, \ldots, 2 k-1$, or $Y_{1,2 k}$. 
In such a way we can able to get $X_{i}\left(Y_{23} Y_{45} Y_{67} \cdots Y_{1,2 k}-Y_{12} Y_{34} \cdots Y_{2 k-1,2 k}\right)$, for all odd $i$, which belongs to $J$, thus $J$ is not a prime ideal.

Now let's show that if $\mathcal{G}$ has at least two connected cycles of odd length, then $S_{R}(I(\mathcal{G}))$ is still not a integrity domain.

Observe that subgraphs, which consist at least of two cycles of odd length with a common edge, contain a cycle of even length.

It remains to examine the case of a subgraph $\mathcal{K}$ of $\mathcal{G}$ on vertex set $[m]=\left\{v_{1}, \ldots\right.$, $\left.v_{h}, \ldots, v_{m}\right\}$ consisting of two cycles of odd lengths $h$ and $m-h+1$ that have $v_{h}$ in common.

Let's consider only the binomials of degree 2 that generate the ideal of relations $J$ of $S_{R}(I(\mathcal{K}))$ and choose the $\frac{m-1}{2}$ ones $X_{i} Y_{i+1, i+2}-X_{i+2} Y_{i, i+1}$, for odd $i=1, \ldots, m-2$, together with $X_{1} Y_{h, m}-X_{m} Y_{1, h}$.

Subtract the last binomial from the sum of the others $\frac{m-1}{2}$, after multiplying each of such $\frac{m+1}{2}$ binomials by suitable $\frac{m-1}{2}$ factors $Y_{j, j+1}, j=1, \ldots, m-1$, or $Y_{1, h}$, or $Y_{h, m}$.

In such a way we get $X_{i}\left(Y_{1, h} Y_{23} Y_{45} \cdots Y_{m-1, m}-Y_{12} Y_{34} \cdots Y_{m-2, m-1} Y_{h, m}\right)$, for all odd $i$, which belongs to $J$, thus $J$ is not a prime ideal.

$\Longleftarrow$ By Proposition 2.7, it is enough to prove that the symmetric powers $S_{t}(I(\mathcal{G}))=R\left[Y_{i j} \mid\left\{v_{i}, v_{j}\right\} \in E(\mathcal{G})\right]_{t} / J_{t}$ are torsion free, $\forall t \geqslant 0$.

Set $B=R\left[Y_{i j} \mid\left\{v_{i}, v_{j}\right\} \in E(\mathcal{G})\right]$. Take $a \in R, a \neq 0$, and $f=\sum_{i} c_{i} f_{i} \in B_{t}$, where $c_{i} \in k$ and the $f_{i}$ are monomials of the same degree in $B_{t}$, such that $a f \in J$.

We want to show that $f \in J$, namely $S_{t}(I(\mathcal{G}))$ has no torsion element. If we consider $\sum_{i} c_{i}=0$, then we can write $f=\sum_{i} c_{i}\left(f_{i}-\bar{f}\right)$, where $\bar{f}$ is a monomial in $B_{t}$ of the same degree of the $f_{i}$. Using the notation of Proposition 2.12 one has

$$
f_{i}-\bar{f}=m \prod_{\left\{v_{i}, v_{j}\right\} \in E_{1}} Y_{i j}-m^{\prime} \prod_{\left\{v_{i^{\prime}}, v_{j^{\prime}}\right\} \in E_{2}} Y_{i^{\prime} j^{\prime}} \in J,
$$

where $E_{1}, E_{2} \subset E(G),\left|E_{1}\right|=\left|E_{2}\right|$, and $m, m^{\prime}$ are monomials in $R$ such that ${ }^{*} \operatorname{supp}(m) \cup \overline{E_{1}}={ }^{*} \operatorname{supp}\left(m^{\prime}\right) \cup \overline{E_{2}}$. It follows by Proposition 2.12 that $f_{i}-\bar{f} \in J$, and so $f \in J$.

\section{Reducedness of the symmetric algebra of edge ideals}

In this section we investigate when the symmetric algebra of the edge ideal of a simple graph is reduced.

Let's give the following characterization for reduced symmetric algebras.

Proposition 3.1. Let $\mathcal{G}$ be a simple graph and $I(\mathcal{G})$ be its edge ideal. The symmetric algebra $S_{R}(I(\mathcal{G}))$ is reduced if and only if $J$ is a radical ideal.

Proof. Let $S_{R}(I(\mathcal{G}))=R\left[Y_{i j} \mid\left\{v_{i}, v_{j}\right\} \in E(\mathcal{G})\right] / J$. According to Remark 1.1, $\operatorname{Nil}\left(S_{R}(I(\mathcal{G}))\right)=\sqrt{\left(0_{S_{R}(I(\mathcal{G}))}\right)}$ and $\sqrt{\left(0_{S_{R}(I(\mathcal{G}))}\right)}=\sqrt{J}$. Then by definition 
$S_{R}(I(\mathcal{G}))$ is reduced if and only if $\operatorname{Nil}\left(S_{R}(I(\mathcal{G}))\right)=\left(0_{S_{R}(I(\mathcal{G}))}\right)$, and this is equivalent to say if and only if $\sqrt{J}=J$, that is $J$ is a radical ideal.

Set $B=R\left[Y_{i j} \mid\left\{v_{i}, v_{j}\right\} \in E(\mathcal{G})\right]$. Let $S_{R}(I(\mathcal{G}))=B / J$ be the symmetric algebra of $I(\mathcal{G})$. Because a domain is reduced, by Theorem 2.13 the symmetric algebra $S_{R}(I(\mathcal{G}))$ is reduced if and only if all the connected subgraphs of $\mathcal{G}$ have either at most a cycle of odd length or no monomial walk.

If $S_{R}(I(\mathcal{G}))$ is not a domain, we investigate its reducedness by using the Gröbner bases theory .

Lemma 3.2. Let $\mathcal{G}$ be a simple graph, $I(\mathcal{G})$ be its edge ideal and $J=\left(g_{1}, \ldots, g_{z}\right)$. Then the S-polynomials $S\left(g_{i}, g_{j}\right)$, for all $i, j=1, \ldots, z$ and $i \neq j$, have the initial term squarefree, for some monomial order $\prec$ on $B$.

Proof. Let $J=\left(g_{1}, \ldots, g_{z}\right) \subset B$ and $\prec$ be a monomial order on $B$. Set $i_{\prec}\left(g_{i}\right)$ the initial term of $g_{i}$ for all $i=1, \ldots, z$. Then the $S$-polynomials of $g_{i}$ and $g_{j}$, for all $i, j=1, \ldots, z$ and $i \neq j$, with respect to $\prec$ are

$$
S\left(g_{i}, g_{j}\right)=\frac{\operatorname{lcm}\left(i n_{\prec}\left(g_{i}\right), i n_{\prec}\left(g_{j}\right)\right)}{i n_{\prec}\left(g_{i}\right)} g_{i}-\frac{\operatorname{lcm}\left(i n_{\prec}\left(g_{i}\right), i n_{\prec}\left(g_{j}\right)\right)}{i n_{\prec}\left(g_{j}\right)} g_{j} .
$$

First observe that if GCD $\left(i_{\prec}\left(g_{i}\right), i_{\prec}\left(g_{j}\right)\right)=1$, then $S\left(g_{i}, g_{j}\right)$ reduces to 0 with respect to $g_{i}, g_{j}$. Hence we consider all the $S$-polynomials $S\left(g_{i}, g_{j}\right)$ such that GCD $\left(i_{\prec}\left(g_{i}\right), i_{\prec}\left(g_{j}\right)\right) \neq 1$ and we show that they have the initial term squarefree, for some monomial order $\prec$ on $B$.

By the structure of the generators of $J$ the following cases occur:

- Let $g_{i}=X_{l} Y_{t k}-X_{t} Y_{l k}, g_{j}=X_{l} Y_{t p}-X_{t} Y_{l p}$, with $\operatorname{in}_{\prec}\left(g_{i}\right)=X_{t} Y_{l k}, \operatorname{in}_{\prec}\left(g_{j}\right)=$ $X_{t} Y_{l p}$. Then $S\left(g_{i}, g_{j}\right)=X_{l} Y_{l p} Y_{t k}-X_{l} Y_{l k} Y_{t p}$.

- Let $g_{i}=X_{l} Y_{t k}-X_{t} Y_{l k}, g_{j}=X_{m} Y_{t p}-X_{t} Y_{m p}$, with $i_{\prec}\left(g_{i}\right)=X_{t} Y_{l k}, i n_{\prec}\left(g_{j}\right)=$ $X_{t} Y_{m p}$. Then $S\left(g_{i}, g_{j}\right)=X_{l} Y_{m p} Y_{t k}-X_{m} Y_{l k} Y_{t p}$.

- Let $g_{i}=X_{l} Y_{t k}-X_{t} Y_{l k}, g_{j}=X_{l} Y_{m k}-X_{m} Y_{l k}$, with $i_{\prec}\left(g_{i}\right)=X_{t} Y_{l k}, i n_{\prec}\left(g_{j}\right)=$ $X_{m} Y_{l k}$. Then $S\left(g_{i}, g_{j}\right)=X_{m} X_{l} Y_{t k}-X_{t} X_{l} Y_{m k}$.

- Let $g_{i}=X_{l} Y_{t k}-X_{t} Y_{l k}, g_{j}=X_{l} X_{k} Y_{p q}-X_{p} X_{q} Y_{l k}$, with $i n_{\prec}\left(g_{i}\right)=X_{t} Y_{l k}, i n_{\prec}\left(g_{j}\right)=$ $X_{p} X_{q} Y_{l k}$. Then $S\left(g_{i}, g_{j}\right)=X_{p} X_{q} X_{l} Y_{l k}-X_{t} X_{l} X_{k} Y_{p q}$.

- Let $g_{i}=X_{l} Y_{t k}-X_{t} Y_{l k}, g_{j}=X_{l} X_{t} Y_{t q}-X_{t} X_{q} Y_{l k}$, with $i n_{\prec}\left(g_{i}\right)=X_{t} Y_{l k}, i_{\prec}\left(g_{j}\right)=$ $X_{t} X_{q} Y_{l k}$. Then $S\left(g_{i}, g_{j}\right)=X_{q} X_{l} Y_{t k}-X_{l} X_{k} Y_{t q}$.

- Let $g_{i}=X_{l} Y_{t k}-X_{t} Y_{l k}, g_{j}=X_{p} X_{m} Y_{t q}-X_{t} X_{q} Y_{p m}$, with $\operatorname{in}_{\prec}\left(g_{i}\right)=X_{t} Y_{l k}, i_{\prec}\left(g_{j}\right)=$ $X_{t} X_{q} Y_{p m}$. Then $S\left(g_{i}, g_{j}\right)=X_{q} X_{l} Y_{t k} Y_{p m}-X_{p} X_{m} Y_{l k} Y_{t q}$.

- Let $g_{i}=X_{l} X_{k} Y_{p k}-X_{p} X_{k} Y_{l t}, g_{j}=X_{q} X_{m} Y_{p k}-X_{p} X_{k} Y_{q m}$, with $i_{\prec}\left(g_{i}\right)=X_{p} X_{k} Y_{l t}$, $i n_{\prec}\left(g_{j}\right)=X_{p} X_{k} Y_{q m}$. Then $S\left(g_{i}, g_{j}\right)=X_{k} X_{l} Y_{p k} Y_{q m}-X_{q} X_{m} Y_{p k} Y_{l t}$.

In all these cases the initial term of $S\left(g_{i}, g_{j}\right)$ is squarefree.

Proposition 3.3. (See [2])

Let $I \subset B$ be an ideal and assume that for some monomial order $\prec$ on $B$ the initial ideal $i_{\prec}(I)$ is generated by squarefree monomials. Then I is a radical ideal. 
Proposition 3.4. Let $\mathcal{G}$ be a simple graph, $I(\mathcal{G})$ be its edge ideal and $J=\left(g_{1}, \ldots, g_{z}\right)$. If the $S$-polynomials $S\left(S\left(g_{i}, g_{j}\right), g_{k}\right)$ and $S\left(S\left(g_{i}, g_{j}\right), S\left(g_{k}, g_{l}\right)\right)$, for all $i, j, k=1, \ldots, z$, $i \neq j$, have a standard expression with respect to $\left\{g_{1}, \ldots, g_{z}, S\left(g_{i}, g_{j}\right), i, j=1, \ldots, z, i \neq\right.$ $j$ \} with remainder 0 , then $S_{R}(I(\mathcal{G}))$ is reduced.

Proof. Let $J=\left(g_{1}, \ldots, g_{z}\right) \subset B, \prec$ be a monomial order on $B$ and $S\left(g_{i}, g_{j}\right)$ be the $S$ polynomials of $g_{i}$ and $g_{j}$, for all $i, j=1, \ldots, z$ and $i \neq j$, with respect to $\prec$. Set $G=$ $\left\{g_{1}, \ldots, g_{z}, S\left(g_{i}, g_{j}\right), i, j=1, \ldots, z, i \neq j\right\}$. If all the $S$-polynomials $S\left(S\left(g_{i}, g_{j}\right), g_{k}\right)$ and $S\left(S\left(g_{i}, g_{j}\right), S\left(g_{k}, g_{l}\right)\right)$ have a standard expression with respect $G$ with remain$\operatorname{der} 0$, for all $i, j, k=1, \ldots, z$ and $i \neq j$, then $G=\left\{g_{1}, \ldots, g_{z}, S\left(g_{i}, g_{j}\right), i, j=1, \ldots, z\right.$, $i \neq j\}$ is a Gröbner basis of $J$. Thus by Lemma 3.2, all the elements of the Gröbner basis of $J$ have squarefree initial terms for some monomial order $\prec$. Hence $i n_{\prec}(J)$ is a squarefree monomial ideal. By Proposition 3.3 J is a radical ideal. The thesis follows from Proposition 3.1 .

Example 3.5. Let $R=k\left[X_{1}, X_{2}, X_{3}, X_{4}\right]$ and consider the graph $\mathcal{C}_{4}$ with edge ideal $I\left(\mathcal{C}_{4}\right)=\left(X_{1} X_{2}, X_{2} X_{3}, X_{3} X_{4}, X_{1} X_{4}\right)$. The ideal of relations of the symmetric algebra $S_{R}\left(I\left(\mathcal{C}_{4}\right)\right)$ is $J=\left(X_{1} Y_{23}-X_{3} Y_{12}, X_{2} Y_{14}-X_{4} Y_{12}, X_{2} Y_{34}-X_{4} Y_{23}, X_{3} Y_{14}-\right.$ $\left.X_{1} Y_{34}\right) \subset R\left[Y_{12}, Y_{23}, Y_{34}, Y_{14}\right]$

Set $g_{1}=X_{1} Y_{23}-X_{3} Y_{12}, g_{2}=X_{2} Y_{14}-X_{4} Y_{12}, g_{3}=X_{2} Y_{34}-X_{4} Y_{23}, g_{4}=X_{3} Y_{14}-$ $X_{1} Y_{34}$. The $S$-polynomials $S\left(g_{1}, g_{2}\right), S\left(g_{1}, g_{3}\right), S\left(g_{1}, g_{4}\right), S\left(g_{2}, g_{4}\right), S\left(g_{3}, g_{4}\right)$ have a standard expression with respect to $\left\{g_{1}, g_{2}, g_{3}, g_{4}\right\}$ with remainder 0 , but $S\left(g_{2}, g_{3}\right)=X_{2} Y_{23} Y_{14}-X_{2} Y_{12} Y_{34}$ does not reduce to 0 with respect to $\left\{g_{1}, g_{2}, g_{3}, g_{4}\right\}$. Let's denote $g_{5}=S\left(g_{2}, g_{3}\right)$.

Set $G=\left\{g_{1}, g_{2}, g_{3}, g_{4}, g_{5}\right\}$. All the $S$-polynomials $S\left(g_{i}, g_{5}\right)$, for $i=1,2,3,4$, have a standard expression with respect to $G$ with remainder 0 . In fact: $S\left(g_{1}, g_{5}\right)=$ $-X_{2} Y_{23} g_{4}, S\left(g_{2}, g_{5}\right)=X_{2} Y_{14} g_{3}, S\left(g_{3}, g_{5}\right)$ reduces to 0 with respect to $G$ because in $\prec\left(g_{3}\right)$, in $\prec\left(g_{5}\right)$ are relatively prime, $S\left(g_{4}, g_{5}\right)=X_{2} Y_{14} g_{1}$. Hence $G$ is a Gröbner basis of $J$ and $i n_{\prec}(J)$ is a squarefree monomial ideal. It follows that $J$ is radical, then $S_{R}(I(\mathcal{G}))$ is reduced.

Remark 3.6. The converse of the Proposition 3.4 is not true in general.

There exist simple graphs for which the symmetric algebra of their edge ideal is reduced, but some $S$-polynomials $S\left(S\left(g_{i}, g_{j}\right), g_{k}\right)$ and $S\left(S\left(g_{i}, g_{j}\right), S\left(g_{k}, g_{l}\right)\right)$ have not a standard expression with respect to $G$ with remainder 0 , for some $i, j, k=$ $1, \ldots, z$ and $i \neq j$. For instance, this occurs to the graph $\mathcal{C}_{6}$.

Next result settles the reducedness of the symmetric algebra of the edge ideals of graphs $\mathcal{C}_{n}$.

Theorem 3.7. Let $\mathcal{C}_{n}$ be the graph with $n$ vertices consisting of a cycle of length $n$. Then the symmetric algebra $S_{R}\left(I\left(\mathcal{C}_{n}\right)\right)$ is reduced.

Proof. Note that, by Theorem 2.13 , the symmetric algebra $S_{R}\left(I\left(\mathcal{C}_{n}\right)\right)$, for $n$ odd, is a integrity domain, and this implies it is reduced.

So it is sufficient to examine the graphs $\mathcal{C}_{n}$, for $n$ even.

Computation is made by using software CoCoA [11].

Let $I\left(\mathcal{C}_{n}\right)=\left(X_{1} X_{2}, X_{2} X_{3}, \ldots X_{n-1} X_{n}, X_{1} X_{n}\right) \subset R=k\left[X_{1}, \ldots, X_{n}\right]$ be the edge ideal of $\mathcal{C}_{n}$. The ideal of relations $J$ of the symmetric algebra $S_{R}\left(I\left(\mathcal{C}_{n}\right)\right)$ is generated by $n$ binomials of degree 2 , 
$X_{2} Y_{1, n}-X_{n} Y_{12}, X_{i} Y_{i+1, i+2}-X_{i+2} Y_{i, i+1}$, for $i=1, \ldots, n-2, X_{n-1} Y_{1, n}-X_{1} Y_{n-1, n}$

and, when $n \geqslant 6$, by $\frac{n(n-5)}{2}$ binomials of degree 3 ,

$X_{3} X_{4} Y_{1, n}-X_{1} X_{n} Y_{34}, \ldots, X_{n-3} X_{n-2} Y_{1, n}-X_{1} X_{n} Y_{n-3, n-2}$

$X_{2} X_{3} Y_{n-1, n}-X_{n-1} X_{n} Y_{23}, \ldots, X_{n-4} X_{n-3} Y_{n-1, n}-X_{n-1} X_{n} Y_{n-4, n-3}$

$X_{1} X_{2} Y_{n-2, n-1}-X_{n-2} X_{n-1} Y_{12}, \ldots, X_{n-5} X_{n-4} Y_{n-2, n-1}-X_{n-2} X_{n-1} Y_{n-5, n-4}$,

$X_{1} X_{2} Y_{n-3, n-2}-X_{n-3} X_{n-2} Y_{12}, \ldots, X_{n-6} X_{n-5} Y_{n-3, n-2}-X_{n-3} X_{n-2} Y_{n-6, n-5}$,

...............

$X_{1} X_{2} Y_{56}-X_{5} X_{6} Y_{12}, X_{2} X_{3} Y_{56}-X_{5} X_{6} Y_{23}$

$X_{1} X_{2} Y_{45}-X_{4} X_{5} Y_{12}$.

A reduced Gröbner basis $G$ of $J$ is a set of binomials including the $n+\frac{n(n-5)}{2}$ generators of $J$ together with:

- for $n=4$, the binomial $X_{4} Y_{23} Y_{14}-X_{4} Y_{12} Y_{34}$;

- for $n>4$ and even, the $n / 2$ binomials

$X_{1} X_{2} Y_{n-1, n}-X_{n-1} X_{n} Y_{12}, X_{4} Y_{23} Y_{1, n}-X_{n} Y_{12} Y_{34}, X_{6} Y_{23} Y_{45} Y_{1, n}-X_{n} Y_{12} Y_{34} Y_{56}$ $X_{8} Y_{23} Y_{45} Y_{67} Y_{1, n}-X_{n} Y_{12} \cdots Y_{78}, \ldots, X_{n} Y_{23} \cdots Y_{n-2, n-1} Y_{1, n}-X_{n} Y_{12} \cdots Y_{n-1, n}$.

It can be seen that all the binomials of $G$ have squarefree initial terms, hence the initial ideal of $J$ is a squarefree monomial ideal. Thus the symmetric algebra $S_{R}\left(I\left(\mathcal{C}_{n}\right)\right)$ is reduced by Propositions 3.3 and 3.1 .

According to the last theorem, we can bring forwards good reasons for assuming

Conjecture 3.8. Let $\mathcal{G}$ be any graph having at least a monomial walk. The symmetric algebra $S_{R}(I(\mathcal{G}))$ is reduced.

\section{References}

[1] M. Barbera, M. Imbesi and M. La Barbiera - On the symmetric algebras associated to graphs with loops, submitted.

[2] V. Ene and J. Herzog - Gröbner bases in Commutative Algebra, Graduate Studies in Mathematics, 130, American Math. Soc. (2011).

[3] E.G. Evans and P. Griffith - Syzygies, London Math. Soc. Lect. Note Ser., 106, Cambridge Univ. Press (1985).

[4] M. Imbesi - Integrality of the symmetric algebra of graph ideals, Math. Notes, 94, 2 (2013), 191-204.

[5] M. Imbesi - Projective dimension and symmetric algebra of graph ideals, Miskolc Math. Notes, 13, 2 (2012), 349-364.

[6] M. Imbesi and M. La Barbiera - Invariants of symmetric algebras associated to graphs, Turk. J. Math., 36, 3 (2012), 345-358.

[7] M. Imbesi and M. La Barbiera - Monomial ideals of graphs with loops, Anal. Ştiinţ. Univ. Al. I Cuza Iaşi (S.N) Mat., 60, 2 (2014), 321-336. 
[8] H. Matsumura - Commutative ring theory, Cambridge Studies in Adv. Math., 8, Cambridge Univ. Press (1986).

[9] A. Micali - Sur les algèbres universelles, Ann. Inst. Fourier, 14, 2 (1964), 33-87.

[10] A. Micali - Algèbres intègres et sans torsion, Bull. Soc. Math. France, 94 (1966), 5-14.

[11] L. Robbiano and CoCoATeam - $\operatorname{CoCoA}$ : a system for doing Computations in Commutative Algebra (2000), Available at http://cocoa.dima.unige.it

[12] R.H. Villarreal - Monomial Algebras, Monographs and Textbooks in Pure and Applied Math., 238, Marcel Dekker, Inc. (2001).

[13] R.H. Villarreal - Rees algebras of edge ideals, Commun. in Algebra, 23, 9 (1995), 3513-3524.

University of Messina,

Department of Civil Engineering and Applied Mathematics,

Via Nuova Panoramica dello Stretto, Contrada Di Dio, 1 - I 98166 Messina, Italy

e-mail: imbesim@unime.it

University of Messina,

Department of Mathematics and Computer Science

Viale F. Stagno d'Alcontres, 31 - I 98166 Messina, Italy

e-mail: monicalb@unime.it 